Check for updates

Cite this: RSC Adv., 2019, 9, 6627

\title{
Design, synthesis and biological activity evaluation of benzoate compounds as local anesthetics
}

\begin{abstract}
Huiying Zou, ab Guangying Chen*ab and Shiyang Zhou (D)*ab
Tetracaine and pramocaine were used as the lead compounds to design benzoate compounds. The combination principle was used to design the target molecule, and the target molecule was modified by bioisostere formation and modification with alkyl groups. In this research, a total of 16 compounds were designed and synthesized. In the process of synthesis, we selected a route with high total yields, mild conditions and simple operation. Three steps were used in the synthesis of the new target compounds, namely, alkylation, esterification and alkylation. The newly designed target compounds were evaluated via surface anesthesia, infiltration anesthesia, block anesthesia and acute toxicity tests. The results of biological activity experiments showed that compounds $4 \mathrm{~d}, 4 \mathrm{~g}, 4 \mathrm{j}, 4 \mathrm{k}, 4 \mathrm{n}$, and $4 \mathrm{o}$ had a good local anesthetic effect, and the results of acute toxicity tests showed that the target compounds had low toxicity.
\end{abstract}

Received 19th January 2019

Accepted 11th February 2019

DOI: $10.1039 / c 9 r a 00476 a$

rsc.li/rsc-advances

cause addiction and some other toxic effects, such as allergic reactivity, tissue stimulation and instability in aqueous solutions. Hence, the clinical application of cocaine was limited. As a result, structural studies and modifications of cocaine have been carried out in the search for better local anesthetics. From studies of the structure of cocaine, it was gradually realized that the removal of the methyl carboxylate group in the structure of cocaine could eliminate addiction. The local anesthetic effect of ethyl-4-aminobenzenecarboxylate (benzocaine) was first confirmed in 1890, and procaine was successfully developed in 1904. The discovery of procaine made people realize the important role of the 4-aminobenzoate structure in local anesthetic drugs and started the study of the 4-aminobenzoate structure $^{22-24}$ (tetracaine) and the development of local anesthetic drugs with amide ${ }^{25,26}$ (lidocaine), aminoketone ${ }^{27}$ (dyclonine) and aminoether ${ }^{28,29}$ (pramocaine) structures (Fig. 1).

In this research, tetracaine and pramocaine were used as the lead compounds to design the target molecules. The lead compounds were integrated into an organic molecule by the combination principle. Then the integrated organic molecule was modified by bioisostere formation and modification with alkyl groups (Fig. 2). A series of novel target compounds with local anesthetic effects were designed. In terms of the structureactivity relationship (SAR) of the drugs, the designed compounds were composed of three basic skeleton structures, namely, a lipophilic section, a link section and a hydrophilic section (Fig. 3). According to the SAR, the target compounds should have a local anesthetic effect. Structural modification of the integrated molecules was performed in order to ensure that the onset time was short, the duration of action was long and the toxic effect was slight. The newly designed benzoate compounds, with 4-aminobenzoic acid (1a) and 4-hydroxybenzoic acid (1b) as starting materials, were synthesized by the

${ }^{a}$ College of Chemistry and Chemical Engineering, Hainan Normal University, Haikou 571158, China.E-mail: chgying123@163.com; zhoushiyang520@126.com

${ }^{b}$ Key Laboratory of Tropical Medicinal Plant Chemistry of Ministry of Education, Hainan Normal University, Haikou 571158, China 


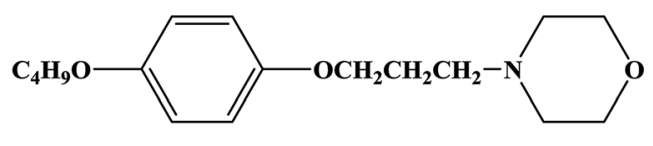

Pramocaine<smiles>CCN(CC)CC(=O)Nc1c(C)cccc1C</smiles>

Lidocaine

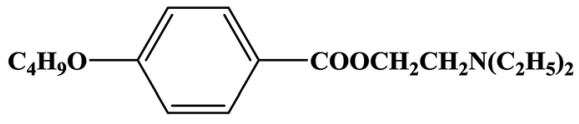

Tetracaine

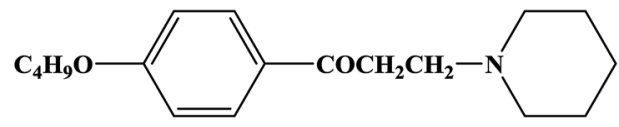

Dyclonine

Fig. 1 Chemical structures of local anesthetics.

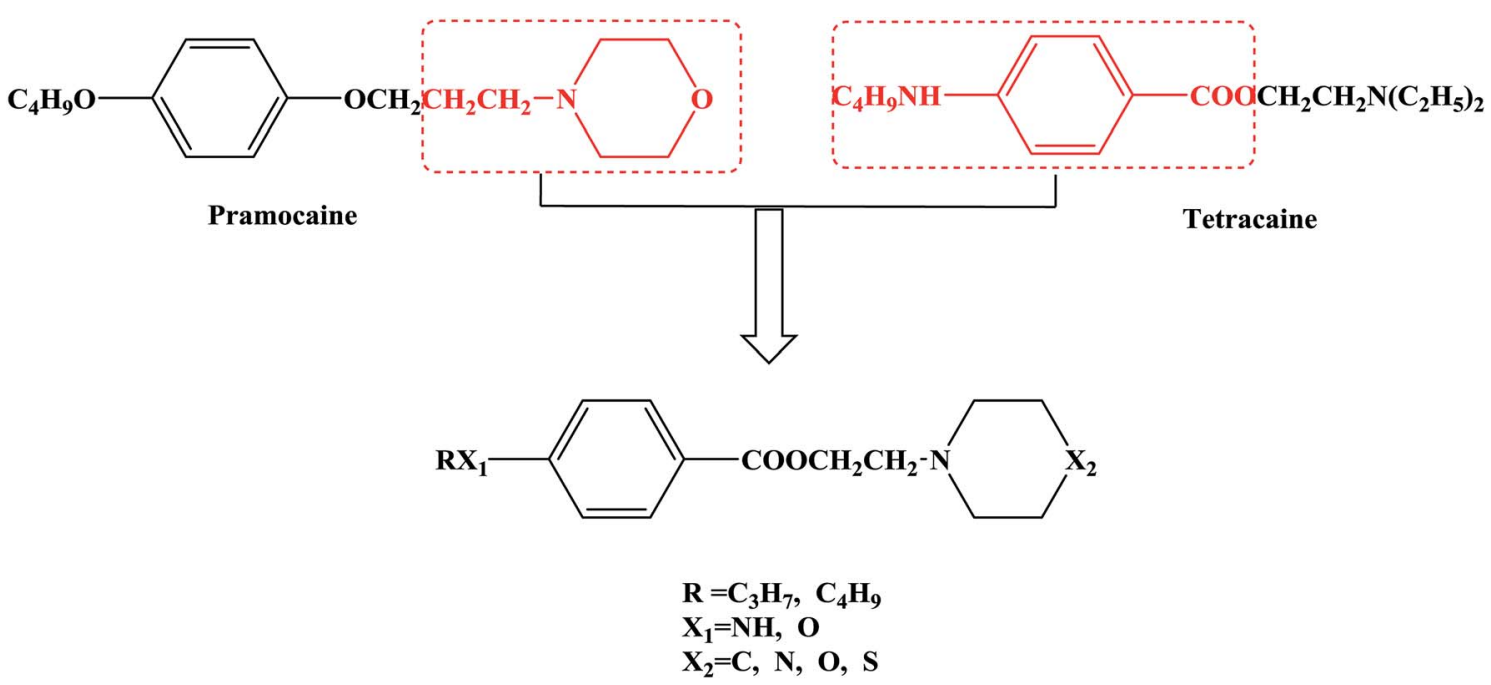

Fig. 2 Design of target compounds.

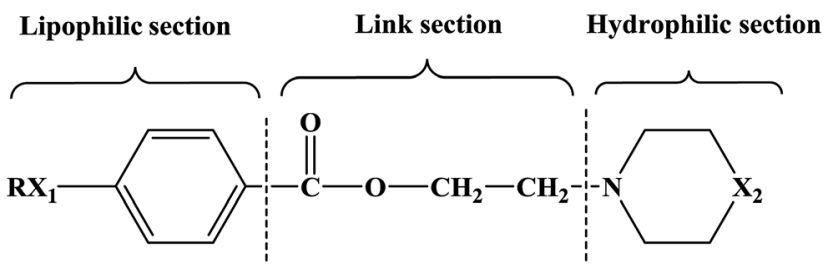

Fig. 3 Basic skeleton structures of target compounds.

three steps of alkylation, esterification and alkylation to give the target compounds (Scheme 1). The synthetic route was characterized by simple operation, high total yields, and mild reaction conditions.

\section{Materials and methods}

\subsection{Chemistry section}

Chemistry materials and general methods. The reagents (chemicals) were purchased and used without further purification. Nuclear magnetic resonance (NMR) spectroscopy was performed using a Bruker AMX-400 spectrometer (IS as TMS). Mass spectroscopy was performed with an Agilent 6460 system. HPLC analysis of all final biologically tested compounds was carried out using an Agilent 1260 series HPLC system. The purity was determined by reversed-phase HPLC and was $\geq 99 \%$ for all biologically tested compounds.

2.1.1. General method for synthesis of compounds $2 a$ and 2b. Firstly, 4-aminobenzoic acid (1a, $13.71 \mathrm{~g}, 0.10 \mathrm{~mol})$ and 1chloropropane $(8.8 \mathrm{~mL}, 0.10 \mathrm{~mol})$ were put into a $500 \mathrm{~mL}$ round-

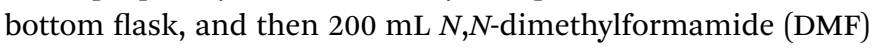
as a solvent, anhydrous sodium carbonate $(5.30 \mathrm{~g}, 0.05 \mathrm{~mol})$ as a deacidifying reagent and $0.1 \%$ of 4 -dimethylaminopyridine (DMAP), which was used as a catalyst for the reaction, were added. After the reactants were added, the reaction lasted for 12 hours under refluxing. When the reflux reaction was completed, the mixture was filtered at a high temperature, the filtrate was collected, cooled, and left to stand for 24 hours, and white crystals were precipitated. The crystals were filtered and dried to obtain the crude product, namely, 4-(propylamino)benzoic acid 


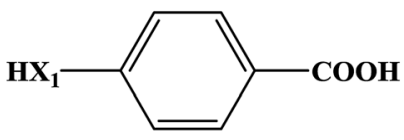

1a-1b

1a: $\mathrm{X}_{1}=\mathrm{NH}$

1b: $X_{1}=O$
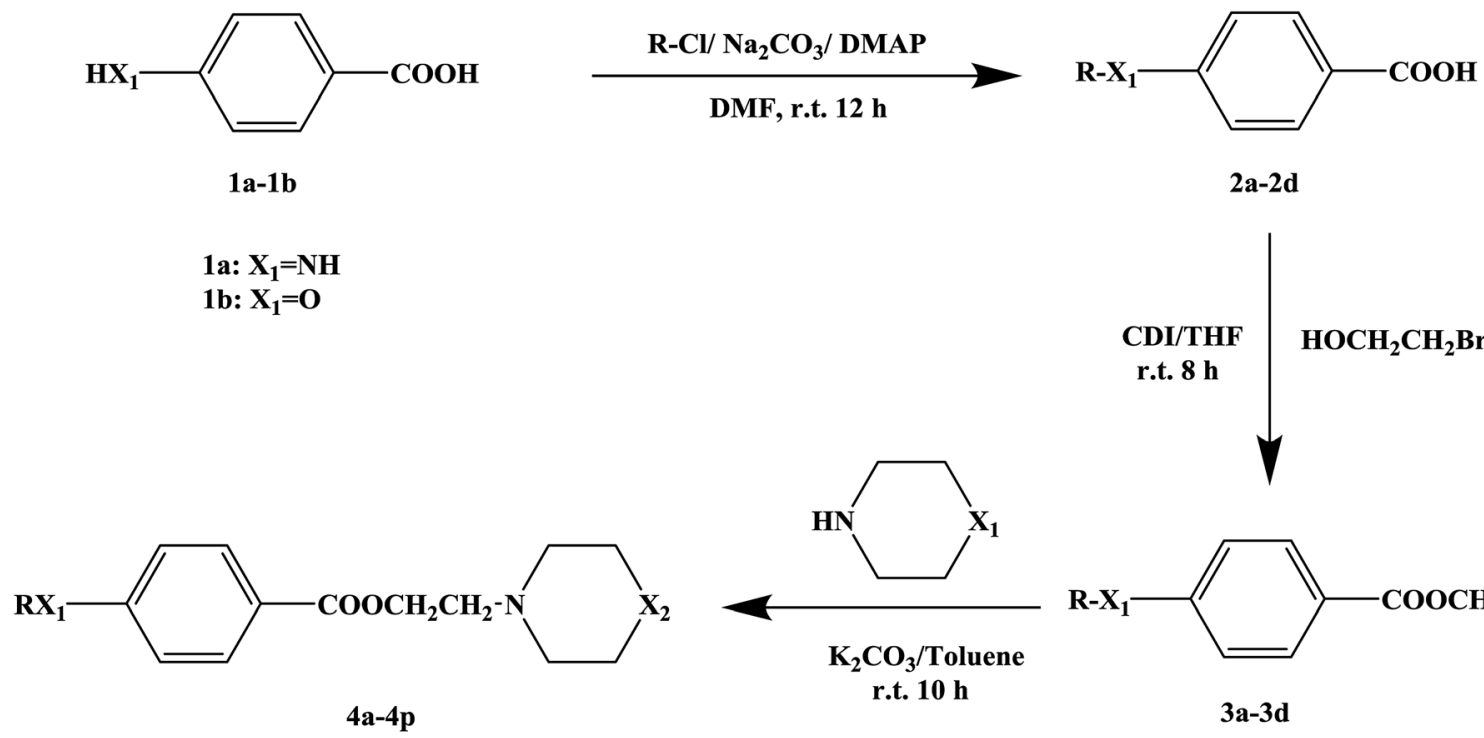

DMF, r.t. 12 h

2a-2d

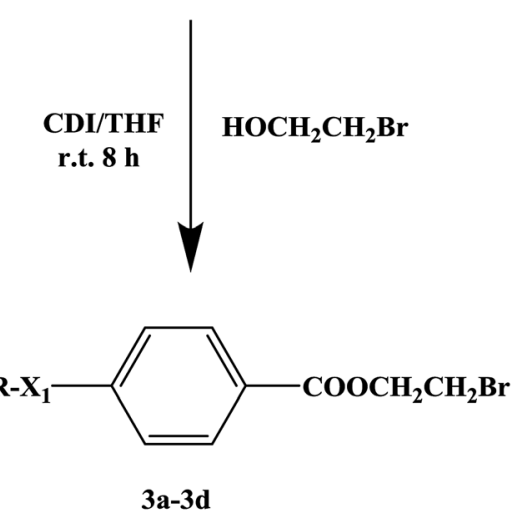

4a: $R=C_{3} H_{7}, X_{1}=N H, X_{2}=C$ 4i: $R=C_{4} H_{9}, X_{1}=N H, X_{2}=C$ 4b: $R=C_{3} H_{7}, X_{1}=N H, X_{2}=N \quad 4 j: R=C_{4} H_{9}, X_{1}=N H, X_{2}=N$ 4c: $R=C_{3} H_{7}, X_{1}=N H, X_{2}=O$ 4k: $R=C_{4} H_{9}, X_{1}=N H, X_{2}=O$ 4d: $R=C_{3} H_{7}, X_{1}=N H, X_{2}=S$ 4l: $R=C_{4} H_{9}, X_{1}=N H, X_{2}=S$ 4e: $R=C_{3} H_{7}, X_{1}=O, \quad X_{2}=C \quad 4 m: R=C_{4} H_{9}, X_{1}=O, \quad X_{2}=C$ 4f: $\mathrm{R}=\mathrm{C}_{3} \mathrm{H}_{7}, \mathrm{X}_{1}=\mathrm{O}, \quad \mathrm{X}_{2}=\mathrm{N} \quad 4 \mathrm{n}: \mathrm{R}=\mathrm{C}_{4} \mathrm{H}_{9}, \mathrm{X}_{1}=\mathrm{O}, \quad \mathrm{X}_{2}=\mathrm{N}$ 4g: $\mathrm{R}=\mathrm{C}_{3} \mathrm{H}_{7}, \mathrm{X}_{1}=\mathrm{O}, \quad \mathrm{X}_{2}=\mathrm{O} \quad$ 4o: $\mathrm{R}=\mathrm{C}_{4} \mathrm{H}_{9}, \mathrm{X}_{1}=\mathrm{O}, \quad \mathrm{X}_{2}=\mathrm{O}$ 4h: $R=C_{3} H_{7}, X_{1}=O, X_{2}=S \quad 4 p: R=C_{4} H_{9}, X_{1}=O, \quad X_{2}=S$

Scheme 1 Synthetic route to target compounds.

(2a). The crude product was recrystallised from toluene, filtered, and dried to give the pure product 4-(propylamino)benzoic acid (2a) as white crystals. The same general method was used to synthesize compound $\mathbf{2 b}$ as white crystals.

2.1.2. General method for synthesis of compounds 3a to 3d. Firstly, $N, N^{\prime}$-carbonyldiimidazole (CDI, $16.22 \mathrm{~g}, 0.10 \mathrm{~mol}$ ) was put into $50 \mathrm{~mL}$ tetrahydrofuran (THF). After CDI was completely dissolved, 4-(propylamino)benzoic acid (2a, $17.92 \mathrm{~g}$, $0.10 \mathrm{~mol}$ ) was added and stirring was continued for 1 hour to form a mixture. The mixture was put into a $500 \mathrm{~mL}$ roundbottom flask, and $150 \mathrm{~mL}$ THF was added. Under reaction conditions of $20^{\circ} \mathrm{C}, 2$-bromoethanamine $(8.7 \mathrm{~mL}, 0.10 \mathrm{~mol})$ was added dropwise. The reaction lasted for 8 hours under refluxing. The mixture was cooled and left to stand for 12 hours, and white crystals were precipitated. The crystals were filtered and vacuum-dried to obtain the crude product, namely, 2bromoethyl-4-(propylamino)benzoate (3a). The crude product was recrystallised from acetone, filtered, and vacuum-dried to give the pure product 2-bromoethyl-4-(propylamino)benzoate (3a) as white crystals. The same general method was used to synthesize compounds $\mathbf{3 b}$ to $\mathbf{3 d}$.

2.1.3. General method for synthesis of compounds 4 a to 4p. Firstly, 2-bromoethyl-4-(propylamino)benzoate (3a, $28.62 \mathrm{~g}$, $0.10 \mathrm{~mol})$ and piperidine $(11.4 \mathrm{~mL}, 0.10 \mathrm{~mol})$ were put into a $500 \mathrm{~mL}$ round-bottom flask, and then $200 \mathrm{~mL}$ toluene as a solvent and anhydrous potassium carbonate $(6.90 \mathrm{~g}, 0.05 \mathrm{~mol})$ as a deacidifying reagent were added. After the reactants were added, the reaction lasted for 8 hours under refluxing. When the reflux reaction was completed, the mixture was filtered at a high temperature, the filtrate was collected, cooled, and left to stand for 12 hours, and white crystals were precipitated. The crystals were filtered and vacuum-dried to obtain the crude product, namely, 2-(piperidin-1-yl)ethyl-4-(propylamino) benzoate (4a). The crude product was recrystallised from acetone, filtered, and vacuum-dried to give the pure product 2(piperidin-1-yl)ethyl-4-(propylamino)benzoate (4a) as white crystals. The same general method was used to synthesize compounds $\mathbf{4 b}$ to $\mathbf{4 p}$.

2-(Piperidin-1-yl)ethyl-4-(propylamino)benzoate $\quad(\mathbf{4 a})$. White crystals, yield $82.1 \%, \mathrm{mp} 141-143{ }^{\circ} \mathrm{C} ;{ }^{1} \mathrm{H}$ NMR $(300 \mathrm{MHz}$, DMSO) $\delta: 0.90\left(3 \mathrm{H}, \mathrm{t}, J=7.1 \mathrm{~Hz},-\mathrm{CH}_{3}\right), 1.38(2 \mathrm{H}, \mathrm{dtt}, J=10.8$, $\left.3.3,2.4 \mathrm{~Hz},-\mathrm{CH}_{2}{ }^{-}\right), 1.50\left(4 \mathrm{H}, \mathrm{ddd}, J=10.8,3.3,2.4 \mathrm{~Hz},-\mathrm{CH}_{2}-\right.$ ), $1.57\left(2 \mathrm{H}, \mathrm{tq}, J=7.4,7.1 \mathrm{~Hz},-\mathrm{CH}_{2}-\right), 2.42(4 \mathrm{H}, \mathrm{ddd}, J=12.5,2.9$, $\left.2.6 \mathrm{~Hz},-\mathrm{CH}_{2}-\right), 3.15\left(2 \mathrm{H}, \mathrm{t}, J=7.1 \mathrm{~Hz},-\mathrm{CH}_{2}-\right), 3.36(2 \mathrm{H}, \mathrm{t}, J=$ $\left.7.4 \mathrm{~Hz},-\mathrm{CH}_{2}-\right), 4.29\left(2 \mathrm{H}, \mathrm{t}, J=7.1 \mathrm{~Hz},-\mathrm{CH}_{2}-\right), 6.79(2 \mathrm{H}, \mathrm{ddd}, J=$ 8.6, 1.2, $0.5 \mathrm{~Hz}, \mathrm{Ph}-\mathrm{H}), 7.69$ (2H, ddd, $J=8.6,1.7,0.5 \mathrm{~Hz}, \mathrm{Ph}-\mathrm{H}$ ); ${ }^{13} \mathrm{C}$ NMR (75 MHz, DMSO) $\delta: 11.4,23.2,24.6,25.9,47.1,54.6$, $56.7,63.1,112.1,118.4,130.6,151.8,165.9$; HR-ESI-MS $\mathrm{m} / \mathrm{z}$ : calcd for $\mathrm{C}_{17} \mathrm{H}_{26} \mathrm{~N}_{2} \mathrm{O}_{2}\left\{[\mathrm{M}+\mathrm{H}]^{+}\right\}$290.4073, found 290.1998; anal. calcd for $\mathrm{C}_{17} \mathrm{H}_{26} \mathrm{~N}_{2} \mathrm{O}_{2}$ : C, 70.31; H, 9.02; N, 9.65; O, 11.02; found: C, 70.33; H, 9.01; N, 9.64; O, 11.02\%.

2-(Piperazin-1-yl)ethyl-4-(propylamino)benzoate $\quad(\mathbf{4} \boldsymbol{b})$. White crystals, yield $86.3 \%$, mp $180-182{ }^{\circ} \mathrm{C} ;{ }^{1} \mathrm{H}$ NMR (300 MHz, DMSO) $\delta: 0.90\left(3 \mathrm{H}, \mathrm{t}, J=7.1 \mathrm{~Hz},-\mathrm{CH}_{3}\right), 1.59(2 \mathrm{H}, \mathrm{tq}, J=7.4,7.1 \mathrm{~Hz}$, $\left.-\mathrm{CH}_{2}{ }^{-}\right), 2.33\left(4 \mathrm{H}, \mathrm{ddd}, J=14.1,10.2,2.5 \mathrm{~Hz},-\mathrm{CH}_{2}{ }^{-}\right), 2.66(4 \mathrm{H}$, $\left.\operatorname{ddd}, J=10.2,9.4,2.5 \mathrm{~Hz},-\mathrm{CH}_{2}-\right), 3.16\left(4 \mathrm{H}, \mathrm{t}, J=7.1 \mathrm{~Hz},-\mathrm{CH}_{2}-\right)$, 
$3.34\left(2 \mathrm{H}, \mathrm{t}, J=7.4 \mathrm{~Hz},-\mathrm{CH}_{2}-\right), 4.31\left(2 \mathrm{H}, \mathrm{t}, J=7.1 \mathrm{~Hz},-\mathrm{CH}_{2}-\right), 6.77$ (2H, ddd, $J=8.6,1.2,0.5 \mathrm{~Hz}, \mathrm{Ph}-\mathrm{H}), 7.69$ (2H, ddd, $J=8.6,1.7$, $0.5 \mathrm{~Hz}, \mathrm{Ph}-\mathrm{H}) ;{ }^{13} \mathrm{C}$ NMR (75 MHz, DMSO) $\delta: 11.4,23.2,46.3,47.3$, 54.3, 57.4, 63.3, 112.0, 118.4, 130.6, 151.8, 165.8; HR-ESI-MS $m / z$ : calcd for $\mathrm{C}_{16} \mathrm{H}_{25} \mathrm{~N}_{3} \mathrm{O}_{2}\left\{[\mathrm{M}+\mathrm{H}]^{+}\right\}$291.3954, found 291.1945; anal. calcd for $\mathrm{C}_{16} \mathrm{H}_{25} \mathrm{~N}_{3} \mathrm{O}_{2}$ : C, 65.95; $\mathrm{H}, 8.65 ; \mathrm{N}, 14.42 ; \mathrm{O}, 10.98$; found: C, 65.96; H, 8.64; N, 14.43; O, 10.99\%.

2-Morpholinoethyl-4-(propylamino)benzoate (4c). White crystals, yield 90.4\%, mp 140-142 ${ }^{\circ} \mathrm{C} ;{ }^{1} \mathrm{H}$ NMR (300 MHz, DMSO) $\delta$ : $0.90\left(3 \mathrm{H}, \mathrm{t}, J=7.1 \mathrm{~Hz},-\mathrm{CH}_{3}\right), 1.57\left(2 \mathrm{H}, \mathrm{tq}, J=7.4,7.1 \mathrm{~Hz},-\mathrm{CH}_{2}-\right.$ ), $2.50\left(4 \mathrm{H}, \mathrm{ddd}, J=9.4,3.1,2.5 \mathrm{~Hz},-\mathrm{CH}_{2}-\right), 3.14(2 \mathrm{H}, \mathrm{t}, J=$ $\left.7.1 \mathrm{~Hz},-\mathrm{CH}_{2}-\right), 3.34\left(2 \mathrm{H}, \mathrm{t}, J=7.4 \mathrm{~Hz},-\mathrm{CH}_{2}-\right), 3.56(4 \mathrm{H}, \mathrm{ddd}, J=$ 11.8, 10.2, $\left.2.5 \mathrm{~Hz},-\mathrm{CH}_{2}-\right), 4.30\left(2 \mathrm{H}, \mathrm{t}, J=7.1 \mathrm{~Hz},-\mathrm{CH}_{2}-\right), 6.77$ $(2 \mathrm{H}, \mathrm{ddd}, J=8.6,1.2,0.5 \mathrm{~Hz}, \mathrm{Ph}-\mathrm{H}), 7.67$ (2H, ddd, $J=8.6,1.7$, $0.5 \mathrm{~Hz}, \mathrm{Ph}-\mathrm{H}) ;{ }^{13} \mathrm{C}$ NMR (75 MHz, DMSO) $\delta: 11.4,23.2,47.3,54.4$, $55.7,63.3$, 66.7, 112.0, 118.4, 130.6, 151.8, 165.8; HR-ESI-MS $m /$ $z$ : calcd for $\mathrm{C}_{16} \mathrm{H}_{24} \mathrm{~N}_{2} \mathrm{O}_{3}\left\{[\mathrm{M}+\mathrm{H}]^{+}\right\}$292.3793, found 292.1789; anal. calcd for $\mathrm{C}_{16} \mathrm{H}_{24} \mathrm{~N}_{2} \mathrm{O}_{3}$ : C, 65.73; H, 8.27; N, 9.58; O, 16.42; found: C, 65.74; H, 8.26; N, 9.59; O, 16.41\%.

2-Thiomorpholinoethyl-4-(propylamino)benzoate (4d). White crystals, yield $85.3 \%$, mp $172-174{ }^{\circ} \mathrm{C} ;{ }^{1} \mathrm{H}$ NMR $(300 \mathrm{MHz}$, DMSO) $\delta: 0.90\left(3 \mathrm{H}, \mathrm{t}, J=7.1 \mathrm{~Hz},-\mathrm{CH}_{3}\right), 1.57(2 \mathrm{H}, \mathrm{tq}, J=7.4$, $\left.7.1 \mathrm{~Hz},-\mathrm{CH}_{2}-\right), 2.55\left(4 \mathrm{H}, \mathrm{ddd}, J=10.9,10.2,2.3 \mathrm{~Hz},-\mathrm{CH}_{2}^{-}\right)$, $2.74\left(4 \mathrm{H}, \mathrm{ddd}, J=8.7,3.4,2.3 \mathrm{~Hz},-\mathrm{CH}_{2}-\right), 3.14(2 \mathrm{H}, \mathrm{t}, J=7.1 \mathrm{~Hz}$, $\left.-\mathrm{CH}_{2}-\right), 3.34\left(2 \mathrm{H}, \mathrm{t}, J=7.4 \mathrm{~Hz},-\mathrm{CH}_{2}-\right), 4.30(2 \mathrm{H}, \mathrm{t}, J=7.1 \mathrm{~Hz}$, $-\mathrm{CH}_{2}-$ ) 6.77 (2H, ddd, $\left.J=8.6,1.2,0.5 \mathrm{~Hz}, \mathrm{Ph}-\mathrm{H}\right), 7.67$ (2H, ddd, $J=8.6,1.7,0.5 \mathrm{~Hz}, \mathrm{Ph}-\mathrm{H}) ;{ }^{13} \mathrm{C}$ NMR (75 MHz, DMSO) $\delta: 11.4$, 23.2, 28.4, 47.3, 53.7, 60.4, 63.3, 112.0, 118.4, 130.6, 151.8, 165.8; HR-ESI-MS $m / z$ : calcd for $\mathrm{C}_{16} \mathrm{H}_{24} \mathrm{~N}_{2} \mathrm{O}_{2} \mathrm{~S}\left\{[\mathrm{M}+\mathrm{H}]^{+}\right\}$308.4403, found 308.1554; anal. calcd for $\mathrm{C}_{16} \mathrm{H}_{24} \mathrm{~N}_{2} \mathrm{O}_{2} \mathrm{~S}$ : C, 62.31; $\mathrm{H}$, 7.84; N, 9.08; O, 10.37; S, 10.39; found: C, 62.33; H, 7.83; N, 9.07; O, 10.38; S, 10.38\%.

2-(Piperidin-1-yl)ethyl-4-propoxybenzoate (4e). White crystals, yield $80.5 \%$, mp $108-110{ }^{\circ} \mathrm{C} ;{ }^{1} \mathrm{H}$ NMR (300 MHz, DMSO) $\delta: 0.98$ $\left(3 \mathrm{H}, \mathrm{t}, J=7.5 \mathrm{~Hz},-\mathrm{CH}_{3}\right), 1.36(2 \mathrm{H}, \mathrm{dtt}, J=10.8,3.3,2.4 \mathrm{~Hz}$, $\left.-\mathrm{CH}_{2}-\right), 1.48\left(4 \mathrm{H}, \mathrm{ddd}, J=10.8,3.3,2.6 \mathrm{~Hz},-\mathrm{CH}_{2}-\right), 1.74(2 \mathrm{H}, \mathrm{t}, J$ $\left.=7.5 \mathrm{~Hz},-\mathrm{CH}_{2}-\right), 2.43\left(4 \mathrm{H}, \mathrm{ddd}, J=12.5,2.9,2.6 \mathrm{~Hz},-\mathrm{CH}_{2}-\right)$, $3.14\left(2 \mathrm{H}, \mathrm{t}, J=7.1 \mathrm{~Hz},-\mathrm{CH}_{2}-\right), 4.02\left(2 \mathrm{H}, \mathrm{t}, J=7.5 \mathrm{~Hz},-\mathrm{CH}_{2}-\right)$, $4.30\left(2 \mathrm{H}, \mathrm{t}, J=7.1 \mathrm{~Hz},-\mathrm{CH}_{2}-\right), 7.06(2 \mathrm{H}, \mathrm{ddd}, J=8.6,1.2,0.4 \mathrm{~Hz}$, Ph-H), 7.95 (2H, ddd, $J=8.6,1.4,0.4 \mathrm{~Hz}, \mathrm{Ph}-\mathrm{H}) ;{ }^{13} \mathrm{C}$ NMR (75 MHz, DMSO) $\delta: 10.5,22.5,24.4,25.8,54.4,56.7,63.3,69.4$, 114.4, 121.6, 130.4, 163.6, 165.8; HR-ESI-MS $\mathrm{m} / \mathrm{z}$ : calcd for $\mathrm{C}_{17} \mathrm{H}_{25} \mathrm{NO}_{3}\left\{[\mathrm{M}+\mathrm{H}]^{+}\right\}$291.3912, found 291.1836; anal. calcd for $\mathrm{C}_{17} \mathrm{H}_{25} \mathrm{NO}_{3}$ : C, 70.07; H, 8.65; N, 4.81; O, 16.47; found: C, 70.06; $\mathrm{H}, 8.66 ; \mathrm{N}, 4.82 ; \mathrm{O}, 16.46 \%$.

2-(Piperazin-1-yl)ethyl-4-propoxybenzoate (4f). White crystals, yield $84.2 \%$, mp $170-172{ }^{\circ} \mathrm{C} ;{ }^{1} \mathrm{H}$ NMR (300 MHz, DMSO) $\delta: 0.98$ $\left(3 \mathrm{H}, \mathrm{t}, J=7.5 \mathrm{~Hz},-\mathrm{CH}_{3}\right), 1.74(2 \mathrm{H}, \mathrm{h}, J=7.5 \mathrm{~Hz}), 2.35(4 \mathrm{H}, \mathrm{ddd}, J$ $\left.=14.1,10.2,2.5 \mathrm{~Hz},-\mathrm{CH}_{2}-\right), 2.64(4 \mathrm{H}, \mathrm{ddd}, J=10.2,9.4,2.5 \mathrm{~Hz}$, $\left.-\mathrm{CH}_{2}-\right), 3.14\left(2 \mathrm{H}, \mathrm{t}, J=7.1 \mathrm{~Hz},-\mathrm{CH}_{2}-\right), 4.02(2 \mathrm{H}, \mathrm{t}, J=7.5 \mathrm{~Hz}$, $\left.-\mathrm{CH}_{2}-\right), 4.30\left(2 \mathrm{H}, \mathrm{t}, J=7.1 \mathrm{~Hz},-\mathrm{CH}_{2}-\right), 7.06(2 \mathrm{H}, \mathrm{ddd}, J=8.6$, 1.2, $0.4 \mathrm{~Hz}, \mathrm{Ph}-\mathrm{H}), 7.95$ (2H, ddd, $J=8.6,1.4,0.4 \mathrm{~Hz}, \mathrm{Ph}-\mathrm{H}) ;{ }^{13} \mathrm{C}$ NMR (75 MHz, DMSO) $\delta: 10.5,22.5,46.3,54.3,57.4,63.3,69.4$, 114.4, 121.6, 130.4, 163.6, 165.8; HR-ESI-MS $\mathrm{m} / \mathrm{z}$ : calcd for $\mathrm{C}_{16} \mathrm{H}_{24} \mathrm{~N}_{2} \mathrm{O}_{3}\left\{[\mathrm{M}+\mathrm{H}]^{+}\right\}$292.3793, found 292.1788; anal. calcd for $\mathrm{C}_{16} \mathrm{H}_{24} \mathrm{~N}_{2} \mathrm{O}_{3}$ : C, 65.73; H, 8.27; N, 9.58; O, 16.42; found: C, 65.74; H, 8.28; N, 9.57; O, 16.41\%.
2-Morpholinoethyl-4-propoxybenzoate (4g). White crystals, yield 88.7\%, mp 113-115 ${ }^{\circ} \mathrm{C} ;{ }^{1} \mathrm{H}$ NMR (300 MHz, DMSO) $\delta: 0.98$ $\left(3 \mathrm{H}, \mathrm{t}, J=7.5 \mathrm{~Hz},-\mathrm{CH}_{3}\right), 1.74\left(2 \mathrm{H}, \mathrm{t}, J=7.5 \mathrm{~Hz},-\mathrm{CH}_{2}-\right), 2.50(4 \mathrm{H}$, $\left.\mathrm{ddd}, J=9.4,3.1,2.5 \mathrm{~Hz},-\mathrm{CH}_{2}-\right), 3.14\left(2 \mathrm{H}, \mathrm{t}, J=7.1 \mathrm{~Hz},-\mathrm{CH}_{2}^{-}\right)$, $3.56\left(4 \mathrm{H}\right.$, ddd, $\left.J=11.8,10.2,2.5 \mathrm{~Hz},-\mathrm{CH}_{2}-\right), 4.02(2 \mathrm{H}, \mathrm{t}, J=$ $\left.7.5 \mathrm{~Hz},-\mathrm{CH}_{2}-\right), 4.30\left(2 \mathrm{H}, \mathrm{t}, J=7.1 \mathrm{~Hz},-\mathrm{CH}_{2}-\right), 7.06(2 \mathrm{H}, \mathrm{ddd}, J=$ 8.6, 1.2, 0.4 Hz, Ph-H), 7.95 (2H, ddd, J=8.6, 1.4, 0.4 Hz, Ph-H); ${ }^{13} \mathrm{C}$ NMR (75 MHz, DMSO) $\delta:$ 10.5, 22.5, 54.4, 55.7, 63.3, 66.7, 69.4, 114.4, 121.6, 130.4, 163.6, 165.8; HR-ESI-MS $\mathrm{m} / \mathrm{z}$ : calcd for $\mathrm{C}_{16} \mathrm{H}_{23} \mathrm{NO}_{4}\left\{[\mathrm{M}+\mathrm{H}]^{+}\right\}$293.3631, found 293.1629; anal. calcd for $\mathrm{C}_{16} \mathrm{H}_{23} \mathrm{NO}_{4}$ : C, 65.51; H, 7.90; N, 4.77; O, 21.81; found: C, 65.52; $\mathrm{H}, 7.91$; N, 4.76; O, 21.80\%.

2-Thiomorpholinoethyl-4-propoxybenzoate (4h). White crystals, yield 83.1\%, mp $150-152{ }^{\circ} \mathrm{C} ;{ }^{1} \mathrm{H}$ NMR (300 MHz, DMSO) $\delta: 0.98$ $\left(3 \mathrm{H}, \mathrm{t}, J=7.5 \mathrm{~Hz},-\mathrm{CH}_{3}\right), 1.74\left(2 \mathrm{H}, \mathrm{t}, J=7.5 \mathrm{~Hz},-\mathrm{CH}_{2}-\right), 2.55(4 \mathrm{H}$, ddd, $\left.J=10.9,3.4,2.2 \mathrm{~Hz},-\mathrm{CH}_{2}-\right), 2.74(4 \mathrm{H}$, ddd, $J=10.2$, 8.7, $\left.2.2 \mathrm{~Hz},-\mathrm{CH}_{2}-\right), 3.14\left(2 \mathrm{H}, \mathrm{t}, J=7.1 \mathrm{~Hz},-\mathrm{CH}_{2}-\right), 4.02(2 \mathrm{H}, \mathrm{t}, J=$ $\left.7.5 \mathrm{~Hz},-\mathrm{CH}_{2}-\right), 4.30\left(2 \mathrm{H}, \mathrm{t}, J=7.1 \mathrm{~Hz},-\mathrm{CH}_{2}-\right), 7.06(2 \mathrm{H}, \mathrm{ddd}, J=$ 8.6, 1.2, 0.4 Hz, Ph-H), 7.95 (2H, ddd, $J=8.6,1.4,0.4 \mathrm{~Hz}, \mathrm{Ph}-\mathrm{H})$; ${ }^{13} \mathrm{C}$ NMR (75 MHz, DMSO) $\delta: 10.5,22.5,28.4,53.7,60.2,63.3$, 69.4, 114.4, 121.6, 130.4, 163.6, 165.8; HR-ESI-MS $\mathrm{m} / \mathrm{z}$ : calcd for $\mathrm{C}_{16} \mathrm{H}_{23} \mathrm{NO}_{3} \mathrm{~S}\left\{[\mathrm{M}+\mathrm{H}]^{+}\right\}$309.4243, found 309.1401; anal. calcd for $\mathrm{C}_{16} \mathrm{H}_{23} \mathrm{NO}_{3} \mathrm{~S}$ : C, 62.11; H, 7.49; N, 4.53; O, 15.51; S, 10.36; found: C, 62.13; H, 7.48; N, 4.52; O, 15.52; S, 10.35\%.

2-(Piperidin-1-yl)ethyl-4-(butylamino)benzoate (4i). White crystals, yield 76.3\%, mp $148-150{ }^{\circ} \mathrm{C} ;{ }^{1} \mathrm{H}$ NMR (300 MHz, DMSO) $\delta$ : $0.88\left(3 \mathrm{H}, \mathrm{t}, J=7.1 \mathrm{~Hz},-\mathrm{CH}_{3}\right), 1.33\left(2 \mathrm{H}, \mathrm{tq}, J=7.4,7.1 \mathrm{~Hz},-\mathrm{CH}_{2}-\right.$ ), $1.36\left(4 \mathrm{H}, \mathrm{dtt}, J=10.8,3.3,2.4 \mathrm{~Hz},-\mathrm{CH}_{2}-\right), 1.46(2 \mathrm{H}, \mathrm{t}, J=$ $\left.7.4 \mathrm{~Hz},-\mathrm{CH}_{2}-\right), 1.48(4 \mathrm{H}$, ddd, $J=10.8,3.3,2.6 \mathrm{~Hz}), 2.43(4 \mathrm{H}$, ddd, $\left.J=12.5,2.9,2.6 \mathrm{~Hz},-\mathrm{CH}_{2}-\right), 3.14\left(2 \mathrm{H}, \mathrm{t}, J=7.1 \mathrm{~Hz},-\mathrm{CH}_{2}-\right)$, $3.30\left(2 \mathrm{H}, \mathrm{t}, J=7.4 \mathrm{~Hz},-\mathrm{CH}_{2}-\right), 4.30\left(2 \mathrm{H}, \mathrm{t}, J=7.1 \mathrm{~Hz},-\mathrm{CH}_{2}-\right)$, $6.77(2 \mathrm{H}, \mathrm{ddd}, J=8.6,1.2,0.4 \mathrm{~Hz}, \mathrm{Ph}-\mathrm{H}), 7.67$ (2H, ddd, $J=8.6$, 1.4, $0.4 \mathrm{~Hz}, \mathrm{Ph}-\mathrm{H}) ;{ }^{13} \mathrm{C}$ NMR (75 MHz, DMSO) $\delta: 13.7,20.1,24.4$, 25.8, 26.0, 43.5, 54.4, 56.7, 63.3, 112.0, 118.4, 130.6, 151.8, 165.8; HR-ESI-MS $m / z$ : calcd for $\mathrm{C}_{18} \mathrm{H}_{28} \mathrm{~N}_{2} \mathrm{O}_{2}\left\{[\mathrm{M}+\mathrm{H}]^{+}\right\}$304.4341, found 304.2153; anal. calcd for $\mathrm{C}_{18} \mathrm{H}_{28} \mathrm{~N}_{2} \mathrm{O}_{2}$ : C, 71.02; $\mathrm{H}, 9.27 ; \mathrm{N}$, 9.20; O, 10.51; found: C, 71.01; H, 9.28; N, 9.21; O, 10.50\%.

2-(Piperazin-1-yl)ethyl-4-(butylamino)benzoate (4j). White crystals, yield 80.4\%, mp 193-195 ${ }^{\circ} \mathrm{C} ;{ }^{1} \mathrm{H}$ NMR (300 MHz, DMSO) $\delta$ : $0.88\left(3 \mathrm{H}, \mathrm{t}, J=7.1 \mathrm{~Hz},-\mathrm{CH}_{3}\right), 1.33\left(2 \mathrm{H}, \mathrm{tq}, J=7.4,7.1 \mathrm{~Hz},-\mathrm{CH}_{2}-\right.$ ), $1.46\left(2 \mathrm{H}, \mathrm{t}, J=7.4 \mathrm{~Hz},-\mathrm{CH}_{2}-\right), 2.35(4 \mathrm{H}, \mathrm{ddd}, J=14.1,10.2$, $\left.2.5 \mathrm{~Hz},-\mathrm{CH}_{2}-\right), 2.64\left(4 \mathrm{H}, \mathrm{ddd}, J=10.2,9.4,2.5 \mathrm{~Hz},-\mathrm{CH}_{2}-\right), 3.14$ $\left(2 \mathrm{H}, \mathrm{t}, J=7.1 \mathrm{~Hz},-\mathrm{CH}_{2}-\right), 3.30\left(2 \mathrm{H}, \mathrm{t}, J=7.4 \mathrm{~Hz},-\mathrm{CH}_{2}-\right), 4.30$ $\left(2 \mathrm{H}, \mathrm{t}, J=7.1 \mathrm{~Hz},-\mathrm{CH}_{2}-\right), 6.77(2 \mathrm{H}, \mathrm{ddd}, J=8.6,1.2,0.4 \mathrm{~Hz}, \mathrm{Ph}-$ $\mathrm{H}), 7.67(2 \mathrm{H}, \mathrm{ddd}, J=8.6,1.4,0.4 \mathrm{~Hz}, \mathrm{Ph}-\mathrm{H}) ;{ }^{13} \mathrm{C} \mathrm{NMR}(75 \mathrm{MHz}$, DMSO) $\delta: 13.7,20.1,26.0,43.5,46.3,54.3,57.4,63.3,112.0$, 118.4, 130.6, 151.8, 165.8; HR-ESI-MS $m / z$ : calcd for $\mathrm{C}_{17} \mathrm{H}_{27} \mathrm{~N}_{3} \mathrm{O}_{2}$ $\left\{[\mathrm{M}+\mathrm{H}]^{+}\right\}$305.4223, found 305.2105; anal. calcd for $\mathrm{C}_{17} \mathrm{H}_{27} \mathrm{~N}_{3} \mathrm{O}_{2}$ : C, 66.85; H, 8.91; N, 13.76; O, 10.48; found: C, 66.86; H, 8.90; N, 13.75; O, 10.49\%.

2-Morpholinoethyl-4-(butylamino)benzoate (4k). White crystals, yield 86.7\%, mp 152-154 ${ }^{\circ} \mathrm{C} ;{ }^{1} \mathrm{H}$ NMR (300 MHz, DMSO) $\delta: 0.88$ $\left(3 \mathrm{H}, \mathrm{t}, J=7.1 \mathrm{~Hz},-\mathrm{CH}_{3}\right), 1.33\left(2 \mathrm{H}, \mathrm{tq}, J=7.4,7.1 \mathrm{~Hz},-\mathrm{CH}_{2}-\right)$, $1.46\left(2 \mathrm{H}, \mathrm{t}, J=7.4 \mathrm{~Hz},-\mathrm{CH}_{2}-\right), 2.50(4 \mathrm{H}, \mathrm{ddd}, J=9.4,3.1,2.5 \mathrm{~Hz}$, $\left.-\mathrm{CH}_{2}-\right), 3.14\left(2 \mathrm{H}, \mathrm{t}, J=7.1 \mathrm{~Hz},-\mathrm{CH}_{2}-\right), 3.30(2 \mathrm{H}, \mathrm{t}, J=7.4 \mathrm{~Hz}$, $\left.-\mathrm{CH}_{2}{ }^{-}\right), 3.56\left(4 \mathrm{H}, \mathrm{ddd}, J=11.8,3.1,2.5 \mathrm{~Hz},-\mathrm{CH}_{2}{ }^{-}\right), 4.30(2 \mathrm{H}, \mathrm{t}, J$ 
$\left.=7.1 \mathrm{~Hz},-\mathrm{CH}_{2}-\right), 6.77(2 \mathrm{H}, \mathrm{ddd}, J=8.6,1.2,0.4 \mathrm{~Hz}, \mathrm{Ph}-\mathrm{H}), 7.67$ ( $2 \mathrm{H}, \mathrm{ddd}, J=8.6,1.4,0.4 \mathrm{~Hz}, \mathrm{Ph}-\mathrm{H}) ;{ }^{13} \mathrm{C} \mathrm{NMR}$ (75 MHz, DMSO) $\delta: 13.7,20.1,26.0,43.5,54.4,55.7,63.3,66.7,112.0,118.4,130.6$, 151.8, 165.8; HR-ESI-MS $m / z$ : calcd for $\mathrm{C}_{17} \mathrm{H}_{26} \mathrm{~N}_{2} \mathrm{O}_{3}\left\{[\mathrm{M}+\mathrm{H}]^{+}\right\}$ 306.4062, found 306.1944; anal. calcd for $\mathrm{C}_{17} \mathrm{H}_{26} \mathrm{~N}_{2} \mathrm{O}_{3}$ : C, 66.64; H, 8.55; N, 9.14; O, 15.66; found: C, 66.63; H, 8.54; N, 9.15; O, $15.67 \%$.

2-Thiomorpholinoethyl-4-(butylamino)benzoate (4l). White crystals, yield $79.2 \%, \mathrm{mp} 184-186{ }^{\circ} \mathrm{C} ;{ }^{1} \mathrm{H}$ NMR $(300 \mathrm{MHz}$, DMSO) $\delta: 0.88\left(3 \mathrm{H}, \mathrm{t}, J=7.1 \mathrm{~Hz},-\mathrm{CH}_{3}\right), 1.33(2 \mathrm{H}, \mathrm{tq}, J=7.4$, $\left.7.1 \mathrm{~Hz},-\mathrm{CH}_{2}-\right), 1.46\left(2 \mathrm{H}, \mathrm{t}, J=7.4 \mathrm{~Hz},-\mathrm{CH}_{2}-\right), 2.55(4 \mathrm{H}, \mathrm{ddd}, J=$ 10.9, 3.4, $\left.2.2 \mathrm{~Hz},-\mathrm{CH}_{2}-\right), 2.74(4 \mathrm{H}$, ddd, $J=8.7,3.4,2.3 \mathrm{~Hz}$, $\left.-\mathrm{CH}_{2}-\right), 3.14\left(2 \mathrm{H}, \mathrm{t}, J=7.1 \mathrm{~Hz},-\mathrm{CH}_{2}-\right), 3.30(2 \mathrm{H}, \mathrm{t}, J=7.4 \mathrm{~Hz}$, $\left.-\mathrm{CH}_{2}-\right), 4.30\left(2 \mathrm{H}, \mathrm{t}, J=7.1 \mathrm{~Hz},-\mathrm{CH}_{2}-\right), 6.77(2 \mathrm{H}, \mathrm{ddd}, J=8.6$, 1.2, 0.4 Hz, Ph-H), 7.67 (2H, ddd, $J=8.6,1.4,0.4 \mathrm{~Hz}, \mathrm{Ph}-\mathrm{H}) ;{ }^{13} \mathrm{C}$ NMR (75 MHz, DMSO) $\delta: 13.7,20.1,26.0,28.4,43.5,53.7,60.4$, $63.3,112.0,118.4,130.6,151.8,165.8$; HR-ESI-MS $\mathrm{m} / \mathrm{z}$ : calcd for $\mathrm{C}_{17} \mathrm{H}_{26} \mathrm{~N}_{2} \mathrm{O}_{2} \mathrm{~S}\left\{[\mathrm{M}+\mathrm{H}]^{+}\right\}$322.4671, found 306.1944; anal. calcd for $\mathrm{C}_{17} \mathrm{H}_{26} \mathrm{~N}_{2} \mathrm{O}_{2} \mathrm{~S}$ : C, 63.32; H, 8.13; N, 8.69; O, 9.92; S, 9.94; found: C, 63.34; H, 8.12; N, 8.68; O, 9.93; S, 9.93\%.

2-(Piperidin-1-yl)ethyl-4-butoxybenzoate (4m). White crystals, yield 78.2\%, mp $115-117{ }^{\circ} \mathrm{C} ;{ }^{1} \mathrm{H}$ NMR (300 MHz, DMSO) $\delta: 0.95$ $\left(3 \mathrm{H}, \mathrm{t}, J=7.1 \mathrm{~Hz},-\mathrm{CH}_{3}\right), 1.37(4 \mathrm{H}, \mathrm{dtt}, J=10.8,3.3,2.4 \mathrm{~Hz}$, $\left.-\mathrm{CH}_{2}-\right), 1.46\left(2 \mathrm{H}, \mathrm{tq}, J=7.4,7.1 \mathrm{~Hz},-\mathrm{CH}_{2}-\right), 1.49(4 \mathrm{H}$, ddd, $J=$ 10.8, 10.2, 3.3, $\left.2.6 \mathrm{~Hz},-\mathrm{CH}_{2}-\right), 1.75\left(2 \mathrm{H}, \mathrm{t}, J=7.4 \mathrm{~Hz},-\mathrm{CH}_{2}-\right)$, $2.43\left(4 \mathrm{H}\right.$, ddd, $\left.J=12.5,2.9,2.6 \mathrm{~Hz},-\mathrm{CH}_{2}-\right), 3.14(2 \mathrm{H}, \mathrm{t}, J=$ $\left.7.1 \mathrm{~Hz},-\mathrm{CH}_{2}-\right), 4.00\left(2 \mathrm{H}, \mathrm{t}, J=7.4 \mathrm{~Hz},-\mathrm{CH}_{2}-\right), 4.30(2 \mathrm{H}, \mathrm{t}, J=$ $\left.7.1 \mathrm{~Hz},-\mathrm{CH}_{2}-\right), 7.06$ (2H, ddd, $\left.J=8.6,1.2,0.4 \mathrm{~Hz}, \mathrm{Ph}-\mathrm{H}\right), 7.95$ (2H, ddd, $J=8.6,1.4,0.4 \mathrm{~Hz}, \mathrm{Ph}-\mathrm{H}) ;{ }^{13} \mathrm{C}$ NMR (75 MHz, DMSO) $\delta: 14.2,19.0,24.4,25.8,31.7,54.4,56.7,63.3,68.5,114.4,121.6$, 130.4, 163.6, 165.8; HR-ESI-MS $m / z$ : calcd for $\mathrm{C}_{18} \mathrm{H}_{27} \mathrm{NO}_{3}\{[\mathrm{M}+$ $\left.\mathrm{H}]^{+}\right\}$305.4183, found 305.1992; anal. calcd for $\mathrm{C}_{18} \mathrm{H}_{27} \mathrm{NO}_{3}$ : C, 70.79; H, 8.91; N, 4.59; O, 15.72; found: C, 70.78; H, 8.92; N, 4.58; O, $15.73 \%$.

2-(Piperazin-1-yl)ethyl-4-butoxybenzoate (4n). White crystals, yield $80.6 \%$, mp $183-185{ }^{\circ} \mathrm{C}$; ${ }^{1} \mathrm{H}$ NMR (300 MHz, DMSO) $\delta: 0.95$ $\left(3 \mathrm{H}, \mathrm{t}, J=7.1 \mathrm{~Hz},-\mathrm{CH}_{3}\right), 1.46\left(2 \mathrm{H}, \mathrm{tq}, J=7.4,7.1 \mathrm{~Hz},-\mathrm{CH}_{2}-\right)$, $1.75\left(2 \mathrm{H}, \mathrm{t}, J=7.4 \mathrm{~Hz},-\mathrm{CH}_{2}\right), 2.35(4 \mathrm{H}, \mathrm{ddd}, J=14.1,10.2$, $\left.2.5 \mathrm{~Hz},-\mathrm{CH}_{2}\right), 2.64\left(\mathrm{ddd}, J=10.2,9.4,2.5 \mathrm{~Hz},-\mathrm{CH}_{2}\right), 3.14(2 \mathrm{H}, \mathrm{t}$, $\left.J=7.1 \mathrm{~Hz},-\mathrm{CH}_{2}\right), 4.00\left(2 \mathrm{H}, \mathrm{t}, J=7.4 \mathrm{~Hz},-\mathrm{CH}_{2}\right), 4.30(2 \mathrm{H}, \mathrm{t}, J=$ $7.1 \mathrm{~Hz},-\mathrm{CH}_{2}$ ), 7.06 (2H, ddd, $\left.J=8.6,1.2,0.4 \mathrm{~Hz}, \mathrm{Ph}-\mathrm{H}\right), 7.95$ ( $2 \mathrm{H}, \mathrm{ddd}, J=8.6,1.4,0.4 \mathrm{~Hz}, \mathrm{Ph}-\mathrm{H}) ;{ }^{13} \mathrm{C}$ NMR (75 MHz, DMSO) $\delta: 14.2,19.0,31.7,46.3,54.3,57.4,63.3,68.5,114.4,121.6,130.4$, 163.6, 165.8; HR-ESI-MS $m / z$ : calcd for $\mathrm{C}_{17} \mathrm{H}_{26} \mathrm{~N}_{2} \mathrm{O}_{3}\left\{[\mathrm{M}+\mathrm{H}]^{+}\right\}$ 306.4065, found 306.1947; anal. calcd for $\mathrm{C}_{17} \mathrm{H}_{26} \mathrm{~N}_{2} \mathrm{O}_{3}$ : C, 66.64; $\mathrm{H}, 8.55$; N, 9.14; O, 15.66; found: C, 66.65; H, 8.54; N, 9.13; O, $15.67 \%$.

2-Morpholinoethyl-4-butoxybenzoate (4o). White crystals, yield 85.5\%, mp 120-122 ${ }^{\circ} \mathrm{C} ;{ }^{1} \mathrm{H}$ NMR (300 MHz, DMSO) $\delta: 0.95(3 \mathrm{H}, \mathrm{t}$, $\left.J=7.1 \mathrm{~Hz},-\mathrm{CH}_{3}\right), 1.46\left(2 \mathrm{H}, \mathrm{tq}, J=7.4,7.1 \mathrm{~Hz},-\mathrm{CH}_{2}-\right), 1.75(2 \mathrm{H}$, $\left.\mathrm{t}, J=7.4 \mathrm{~Hz},-\mathrm{CH}_{2}-\right), 2.50\left(4 \mathrm{H}, \mathrm{ddd}, J=9.4,3.1,2.5 \mathrm{~Hz},-\mathrm{CH}_{2}-\right)$, $3.14\left(2 \mathrm{H}, \mathrm{t}, J=7.1 \mathrm{~Hz},-\mathrm{CH}_{2}-\right), 3.56(4 \mathrm{H}$, ddd, $J=11.8,3.1$, $\left.2.5 \mathrm{~Hz},-\mathrm{CH}_{2}-\right), 4.00\left(2 \mathrm{H}, \mathrm{t}, J=7.4 \mathrm{~Hz},-\mathrm{CH}_{2}-\right), 4.30(2 \mathrm{H}, \mathrm{t}, J=$ $\left.7.1 \mathrm{~Hz},-\mathrm{CH}_{2}-\right)$, 7.06 (2H, ddd, $\left.J=8.6,1.2,0.4 \mathrm{~Hz}, \mathrm{Ph}-\mathrm{H}\right), 7.95$ $(2 \mathrm{H}, \mathrm{ddd}, J=8.6,1.4,0.4 \mathrm{~Hz}, \mathrm{Ph}-\mathrm{H}) ;{ }^{13} \mathrm{C} \mathrm{NMR}(75 \mathrm{MHz}, \mathrm{DMSO})$ $\delta: 14.2,19.0,31.7,54.4,55.6,63.3,66.7,68.5,114.4,121.6,130.4$, 163.6, 165.8; HR-ESI-MS $m / z$ : calcd for $\mathrm{C}_{17} \mathrm{H}_{25} \mathrm{NO}_{4}\left\{[\mathrm{M}+\mathrm{H}]^{+}\right\}$
307.3902, found 307.1787; anal. calcd for $\mathrm{C}_{17} \mathrm{H}_{25} \mathrm{NO}_{4}$ : C, 66.43; H, 8.20; N, 4.56; O, 20.82; found: C, 66.41; H, 8.20; N, 4.57; O, $20.83 \%$.

2-Thiomorpholinoethyl-4-butoxybenzoate (4p). White crystals, yield 80.1\%, mp 162-164 ${ }^{\circ} \mathrm{C} ;{ }^{1} \mathrm{H}$ NMR (300 MHz, DMSO) $\delta: 0.95$ $\left(3 \mathrm{H}, \mathrm{t}, J=7.1 \mathrm{~Hz},-\mathrm{CH}_{3}\right), 1.46\left(2 \mathrm{H}, \mathrm{tq}, J=7.4,7.1 \mathrm{~Hz},-\mathrm{CH}_{2}-\right)$, $1.74\left(2 \mathrm{H}, \mathrm{t}, J=7.4 \mathrm{~Hz},-\mathrm{CH}_{2}-\right), 2.55(4 \mathrm{H}, \mathrm{ddd}, J=10.9,3.4$, $\left.2.2 \mathrm{~Hz},-\mathrm{CH}_{2}-\right), 2.74\left(4 \mathrm{H}, \mathrm{ddd}, J=8.7,3.4,2.3 \mathrm{~Hz},-\mathrm{CH}_{2}^{-}\right), 3.14$ $\left(2 \mathrm{H}, \mathrm{t}, J=7.1 \mathrm{~Hz},-\mathrm{CH}_{2}-\right), 4.00\left(2 \mathrm{H}, \mathrm{t}, J=7.4 \mathrm{~Hz},-\mathrm{CH}_{2}-\right), 4.30$ $\left(2 \mathrm{H}, \mathrm{t}, J=7.1 \mathrm{~Hz},-\mathrm{CH}_{2}-\right), 7.06(2 \mathrm{H}, \mathrm{ddd}, J=8.6,1.2,0.4 \mathrm{~Hz}, \mathrm{Ph}-$ $\mathrm{H}), 7.95(2 \mathrm{H}, \mathrm{ddd}, J=8.6,1.4,0.4 \mathrm{~Hz}, \mathrm{Ph}-\mathrm{H}) ;{ }^{13} \mathrm{C} \mathrm{NMR}(75 \mathrm{MHz}$, DMSO) $\delta: 14.2,19.0,28.4,31.7,53.7,60.4,63.3$, 68.5, 114.4, 121.6, 130.4, 163.6, 165.8; HR-ESI-MS $m / z$ : calcd for $\mathrm{C}_{17} \mathrm{H}_{25} \mathrm{NO}_{3} \mathrm{~S}$ $\left\{[\mathrm{M}+\mathrm{H}]^{+}\right\}$323.4513, found 323.1558; anal. calcd for $\mathrm{C}_{17} \mathrm{H}_{25} \mathrm{NO}_{3} \mathrm{~S}$ : C, 63.13; H, 7.79; N, 4.33; O, 14.84; S, 9.91; found: C, 63.15; H, 7.78; N, 4.32; O, 14.85; S, 9.90\%.

\subsection{Biological section}

2.2.1. Surface anesthesia and invasive anesthesia. The surface anesthetic effects of the target compounds were investigated by corneal reflex experiments in rabbits, and the infiltration anesthetic effects of the target compounds were investigated by abdominal acupuncture experiments in mice.

Surface anesthesia. 190 healthy rabbits without eye diseases were taken and the eyelashes were cut off, and the rabbits were then randomly divided into an average of 19 groups. For each target compound, the rabbits were divided into one experimental group, two positive control groups and one blank control group. Each rabbit's lower eyelid was pulled into a cup shape with the thumb and forefinger, the middle finger was pressed against the nasolacrimal duct to prevent the liquid from flowing into the nasolacrimal duct, and a solution of the corresponding drugs (1\% target compound and $1 \%$ positive control substance) was dropped, with two drops in each eye. The lower eyelid was gently rubbed to allow the drug solution to fully contact the cornea, and it was allowed to overflow after $2 \mathrm{~min}$. Tetracaine and pramocaine were used as the positive controls and NS as the blank control. The effects of surface anesthesia were evaluated by a number of corneal stimulations. Corneal stimulation was used to gently touch the cornea at around 5 points in the upper, lower, middle, left and right parts. After stimulation had been performed 100 times, the blink reflex occurred less than 5 times, and the anesthetic effect was considered to be effective.

The corneal reflex was tested every $10 \mathrm{~min}$ for 1 hour before the completion of the infusion and every $20 \mathrm{~min}$ for 1 hour afterward, and the tests were continued until the blink reflex occurred more than 5 times. Blinks were compared between groups and in terms of how they changed over time.

Invasive anesthesia. 90 healthy mice were taken and randomly divided into 9 groups. For each target compound, the mice were divided into one experimental group, two positive control groups and one blank control group. The hair on the abdominal skin of the mice was shaved in an area with a diameter of about $2.5 \mathrm{~cm}$. A circle with a diameter of $2 \mathrm{~cm}$ was drawn in this area with a marker, and a solution of the corresponding drugs (1\% target compound and $1 \%$ positive control substance) was 
injected, of which $1 \mathrm{~mL}$ was injected subcutaneously to form a pichu. Tetracaine and pramocaine were used as the positive controls and NS as the blank control. A home-made test needle was used to test the response of the skin to the pinprick after the injection. In the beginning, it was tested every 15 min 10 times for each acupuncture and $\mathbf{1 . 5}$ hours after the injection, and the tests were performed every 0.5 hours until the mice had completely recovered to a state without anesthesia. The skin contraction reaction of the mice during acupuncture was positive (no pain inhibition). The changes in the pain inhibition rate over time were compared in each group.

2.2.2. Block anesthesia and acute toxicity tests. The nerve block anesthetic effects of the compounds were evaluated by a rat model of a tail nerve block, and the acute toxicity was evaluated by oral administration to mice.

Block anesthesia. A certain number of rats were taken and subjected to thermal irradiation, and the normal tail flick reaction time of the rats was routinely measured. Eighty rats with a tail length of $2-4 \mathrm{~cm}$ were randomly divided into 8 groups. The radiation intensity of the pain-inducing tail flick instrument was adjusted to 75, and then the rats were fixed and only the rats' tails were exposed. Five points were selected in each tail, and a solution of the corresponding drugs (1\% target compound and $1 \%$ positive control substance) was injected, of which $0.2 \mathrm{~mL}$ was injected at each point (no injection into the blood vessels was allowed). Tetracaine and pramocaine were used as the positive controls and NS as the blank control. After the tail nerve block operation was completed, the length of tail flicks was measured every 2 min with the pain-inducing tail flick instrument, and the onset of analgesia and duration of analgesia were recorded.

Acute toxicity tests. An orally administered Korbor test was performed.

\section{3. $\log P$ and $\mathrm{pK}_{\mathrm{a}}$ tests}

Firstly, an oil phase (octanol) and a water phase were saturated with each other for 24 hours. Then an appropriate amount of the respective compound was added to a test tube, followed by $1 \mathrm{~mL}$ of the pre-saturated two-phase solution. The mouth of the test tube was closed and the test tube was oscillated for 72 hours to make the distribution reach equilibrium. After the distribution was balanced, centrifugal separation was carried out to measure the sample concentrations in the two respective phases, and then $\log P$ was calculated. The compound was also prepared as a dilute solution with a certain concentration, and half of the equivalent sodium hydroxide solution was added. Then, after the solution had been shaken well, the $\mathrm{pH}$ of the solution was directly measured with a $\mathrm{pH}$ meter, and then the $\mathrm{p} K_{\mathrm{a}}$ was calculated.

\section{Results and discussion}

\subsection{Synthesis}

At present, many types of local anesthetic drugs are used in clinical practice, such as tetracaine, lidocaine, dyclonine and pramocaine (Fig. 1). However, these local anesthetic drugs have many deficiencies; for example, tetracaine causes adverse reactions (toxicity), and an excessive dosage of drugs is likely to cause toxic reactions in the central nervous system (CNS) and cardiovascular system. In addition, pramocaine has an anesthetic effect of limited duration. In terms of their chemical structure, these local anesthetic drugs all contain a benzene ring, which is the parent structure (pharmacophore) of this kind of drug. In this research, the target compounds were designed with tetracaine and pramocaine as the lead compounds, and a new organic molecule was formed by the combination principle (Fig. 2). On this basis, the structure of the new organic molecule was modified, and a series of novel benzeneamide compounds were designed by the use of bioisostere formation and modification of the compound with alkyl groups (Fig. 2). The structure of the new organic molecule was determined, the substituent $\mathrm{R}$ on the benzene ring was modified, and two substituents, namely, $-\mathrm{C}_{3} \mathrm{H}_{7}$ and $-\mathrm{C}_{4} \mathrm{H}_{9}$, were selected in the designed process. For $\mathrm{X}_{1}, \mathrm{~N}$ and $\mathrm{O}$ were selected as the connecting atoms. In addition, in the process of modification of the six-membered heterocyclic ring, for $\mathrm{X}_{2} \mathrm{C}, \mathrm{N}, \mathrm{O}$ and $\mathrm{S}$ atoms were selected. From the perspective of the structure-activity relationship (SAR), the newly designed series of compounds had three basic skeleton structures, namely, a lipophilic section, a link section and a hydrophilic section (Fig. 3). The purpose of the modification of $\mathrm{R}$ and $\mathrm{X}_{1}$ was to design target compounds with rapid onset and long duration of anesthesia (lipophilic section), whereas the modification of $\mathrm{X}_{2}$ was performed to obtain compounds with low toxicity (hydrophilic section). In the process of synthesizing the target compounds, we selected a route with high total yields, mild conditions and simple operation. Three steps were used in the synthesis of the new target compounds, namely, alkylation, esterification and alkylation. The first alkylation step used 4-(propylamino)benzoic acid (2a), DMF as a solvent, anhydrous sodium carbonate as a deacidifying reagent and DMAP as a catalyst, and the reflux reaction was completed in 12 hours. The esterification used 2bromoethyl-4-(propylamino)benzoate (3a), THF as a solvent, and CDI as a dehydrating agent, and the reflux reaction was completed in 8 hours. The second alkylation step used 2(piperidin-1-yl)ethyl-4-(propylamino)benzoate (4a), toluene as a solvent, and anhydrous potassium carbonate $\left(\mathrm{K}_{2} \mathrm{CO}_{3}\right)$ as a deacidifying reagent, and the reflux reaction was completed in 10 hours. The products were analyzed by ${ }^{1} \mathrm{H}$ NMR, ${ }^{13} \mathrm{C} \mathrm{NMR}$, MS, and elemental analysis. Moreover, some physical and chemical properties $\left(\log P\right.$ and $\left.\mathrm{p} K_{\mathrm{a}}\right)$ of the compounds were studied (Table 1). From Table 1, we can find that these target compounds had high lipid solubility $(\log P$ ranged from 1.57 to 3.73) and were easily absorbed.

\subsection{Biological activity}

Local anesthetics act on nerve endings and nerve trunks and play a role in local anesthesia. Local anesthetics are commonly used in stomatology, ophthalmology, gynecology and minor surgical operations. The mechanism of action of a local anesthetic is that the drug binds to the receptor of the sodium ion $\left(\mathrm{Na}^{+}\right)$channel on the nerve membrane, reduces the passage of 
Table 1 Physical and chemical properties of the target compounds

\begin{tabular}{|c|c|c|c|c|c|}
\hline Compound & $\mathrm{R}$ & $\mathrm{X}_{1}$ & $\mathrm{X}_{2}$ & $\log P$ & $\mathrm{p} K_{\mathrm{a}}$ \\
\hline $4 a$ & $\mathrm{C}_{3} \mathrm{H}_{7-}$ & $\mathrm{NH}$ & C & 2.93 & 8.79 \\
\hline $4 b$ & $\mathrm{C}_{3} \mathrm{H}_{7-}$ & $\mathrm{NH}$ & $\mathrm{N}$ & 1.57 & 7.90 \\
\hline $4 \mathrm{c}$ & $\mathrm{C}_{3} \mathrm{H}_{7-}$ & $\mathrm{NH}$ & $\mathrm{O}$ & 1.78 & 7.76 \\
\hline 4d & $\mathrm{C}_{3} \mathrm{H}_{7^{-}}$ & $\mathrm{NH}$ & $S$ & 2.54 & 8.00 \\
\hline $4 e$ & $\mathrm{C}_{3} \mathrm{H}_{7-}$ & $\mathrm{O}$ & $\mathrm{C}$ & 3.29 & 8.77 \\
\hline 4f & $\mathrm{C}_{3} \mathrm{H}_{7^{-}}$ & $\mathrm{O}$ & $\mathrm{N}$ & 1.96 & 7.86 \\
\hline $4 g$ & $\mathrm{C}_{3} \mathrm{H}_{7}^{-}$ & $\mathrm{O}$ & $\mathrm{O}$ & 2.18 & 7.73 \\
\hline $4 \mathrm{~h}$ & $\mathrm{C}_{3} \mathrm{H}_{7}^{-}$ & $\mathrm{O}$ & $\mathrm{S}$ & 2.90 & 7.92 \\
\hline $4 i$ & $\mathrm{C}_{4} \mathrm{H}_{9^{-}}$ & $\mathrm{NH}$ & $\mathrm{C}$ & 3.26 & 8.81 \\
\hline $4 \mathbf{j}$ & $\mathrm{C}_{4} \mathrm{H}_{9^{-}}$ & $\mathrm{NH}$ & $\mathrm{N}$ & 1.98 & 7.93 \\
\hline $4 k$ & $\mathrm{C}_{4} \mathrm{H}_{9^{-}}$ & $\mathrm{NH}$ & $\mathrm{O}$ & 2.21 & 7.76 \\
\hline 41 & $\mathrm{C}_{4} \mathrm{H}_{9^{-}}$ & $\mathrm{NH}$ & S & 2.94 & 7.98 \\
\hline $4 m$ & $\mathrm{C}_{4} \mathrm{H}_{9^{-}}$ & $\mathrm{O}$ & $\mathrm{C}$ & 3.73 & 8.76 \\
\hline $4 n$ & $\mathrm{C}_{4} \mathrm{H}_{9^{-}}$ & $\mathrm{O}$ & $\mathrm{N}$ & 2.36 & 7.85 \\
\hline 40 & $\mathrm{C}_{4} \mathrm{H}_{9^{-}}$ & $\mathrm{O}$ & $\mathrm{O}$ & 2.59 & 7.75 \\
\hline $4 p$ & $\mathrm{C}_{4} \mathrm{H}_{9^{-}}$ & $\mathrm{O}$ & $S$ & 3.31 & 7.95 \\
\hline
\end{tabular}

$\mathrm{Na}^{+}$through the ion channel and blocks the conduction of nerve impulses so as to achieve the purpose of local anesthesia. People expect that local anesthetics should have the characteristics of rapid onset, long duration and low toxicity. At present, the commonly used local anesthetics have some disadvantages, such as slow onset, short duration and high toxicity, and hence it is necessary to design new local anesthetics. The newly designed target compounds were evaluated via surface anesthesia, infiltration anesthesia, block anesthesia and acute toxicity tests to select target compounds with good biological activity. The mechanism of action of these compounds was studied. The results of the biological activity tests showed that these compounds had good local anesthetic effects (Tables 2-4). In particular, compounds $\mathbf{4 d}, \mathbf{4 g}, \mathbf{4 j}, \mathbf{4 k}, \mathbf{4 n}$, and $\mathbf{4 o}$ had excellent effects in terms of surface anesthesia, infiltration anesthesia and block anesthesia. In the process of the local anesthesia experiment, it was found that the anesthetic effects of

Table 2 Surface anesthetic effects of the target compounds

\begin{tabular}{|c|c|c|c|c|c|c|c|c|c|c|}
\hline \multirow[b]{2}{*}{ Compound } & \multicolumn{10}{|c|}{ Blink reflexes $(n=10)$} \\
\hline & $10 \mathrm{~min}$ & $20 \mathrm{~min}$ & $30 \min$ & $40 \mathrm{~min}$ & $50 \mathrm{~min}$ & $60 \mathrm{~min}$ & $80 \mathrm{~min}$ & $100 \mathrm{~min}$ & $120 \mathrm{~min}$ & $140 \mathrm{~min}$ \\
\hline $4 \mathrm{~b}$ & $7 \pm 0.4$ & $6 \pm 0.6$ & $5 \pm 0.3$ & $4 \pm 0.3$ & $3 \pm 0.2$ & $3 \pm 0.3$ & $5 \pm 0.5$ & $21 \pm 2.1$ & $40 \pm 4.2$ & $70 \pm 6.2$ \\
\hline $4 \mathrm{c}$ & $6 \pm 0.3$ & $6 \pm 0.7$ & $4 \pm 0.2$ & $3 \pm 0.2$ & $3 \pm 0.2$ & $4 \pm 0.5$ & $6 \pm 0.6$ & $27 \pm 2.5$ & $49 \pm 3.9$ & $89 \pm 7.3$ \\
\hline $4 d$ & $4 \pm 0.3$ & $3 \pm 0.1$ & $3 \pm 0.2$ & $2 \pm 0.1$ & $2 \pm 0.1$ & $2 \pm 0.1$ & $4 \pm 0.5$ & $20 \pm 1.9$ & $39 \pm 4.0$ & $71 \pm 7.3$ \\
\hline $4 \mathrm{~g}$ & $4 \pm 0.2$ & $3 \pm 0.2$ & $2 \pm 0.1$ & $1 \pm 0.1$ & $1 \pm 0.1$ & $2 \pm 0.1$ & $4 \pm 0.7$ & $21 \pm 2.0$ & $38 \pm 2.6$ & $82 \pm 7.5$ \\
\hline $4 \mathrm{~h}$ & $6 \pm 0.3$ & $6 \pm 0.5$ & $5 \pm 0.6$ & $4 \pm 0.5$ & $3 \pm 0.2$ & $3 \pm 0.3$ & $5 \pm 0.7$ & $23 \pm 2.1$ & $41 \pm 4.0$ & $83 \pm 7.6$ \\
\hline $4 \mathrm{i}$ & $7 \pm 0.5$ & $6 \pm 0.7$ & $5 \pm 0.6$ & $4 \pm 0.5$ & $4 \pm 0.5$ & $3 \pm 0.3$ & $6 \pm 0.8$ & $26 \pm 2.2$ & $46 \pm 4.3$ & $86 \pm 8.3$ \\
\hline $4 \mathrm{j}$ & $5 \pm 0.3$ & $4 \pm 0.3$ & $3 \pm 0.2$ & $3 \pm 0.2$ & $2 \pm 0.1$ & $2 \pm 0.1$ & $5 \pm 0.6$ & $24 \pm 2.3$ & $48 \pm 4.1$ & $90 \pm 8.5$ \\
\hline $4 k$ & $4 \pm 0.3$ & $3 \pm 0.2$ & $2 \pm 0.1$ & $1 \pm 0.1$ & $1 \pm 0.1$ & $1 \pm 0.1$ & $3 \pm 0.2$ & $14 \pm 1.3$ & $33 \pm 2.8$ & $69 \pm 5.1$ \\
\hline 41 & $6 \pm 0.6$ & $5 \pm 0.5$ & $4 \pm 0.3$ & $3 \pm 0.2$ & $3 \pm 0.5$ & $4 \pm 0.4$ & $6 \pm 0.7$ & $28 \pm 2.7$ & $50 \pm 4.7$ & $93 \pm 8.9$ \\
\hline Tetracaine & $6 \pm 0.4$ & $5 \pm 0.6$ & $4 \pm 0.4$ & $3 \pm 0.2$ & $2 \pm 0.1$ & $2 \pm 0.1$ & $5 \pm 0.6$ & $18 \pm 1.3$ & $38 \pm 3.0$ & $79 \pm 6.4$ \\
\hline Pramocaine & $6 \pm 0.5$ & $5 \pm 0.8$ & $3 \pm 0.3$ & $2 \pm 0.1$ & $2 \pm 0.1$ & $2 \pm 0.1$ & $6 \pm 0.8$ & $23 \pm 2.1$ & $44 \pm 4.0$ & $89 \pm 7.8$ \\
\hline NS & 100 & 100 & 100 & 100 & 100 & 100 & 100 & 100 & 100 & 100 \\
\hline
\end{tabular}

Table 3 Infiltration anesthetic effects of the target compounds

\begin{tabular}{|c|c|c|c|c|c|c|c|c|c|c|c|c|c|}
\hline \multirow[b]{2}{*}{ Compound } & \multicolumn{13}{|c|}{ Pain inhibition $(n=10, \%)$} \\
\hline & $15 \mathrm{~min}$ & $30 \mathrm{~min}$ & $45 \mathrm{~min}$ & $60 \mathrm{~min}$ & $75 \mathrm{~min}$ & $90 \mathrm{~min}$ & $2 \mathrm{~h}$ & $2.5 \mathrm{~h}$ & $3 \mathrm{~h}$ & $3.5 \mathrm{~h}$ & $4 \mathrm{~h}$ & $4.5 \mathrm{~h}$ & $5 \mathrm{~h}$ \\
\hline $4 \mathrm{~g}$ & 100 & 100 & 100 & 100 & 100 & 100 & 100 & 100 & 100 & 100 & $98.9 \pm 0.2$ & $86.7 \pm 1.2$ & $73.8 \pm 1.4$ \\
\hline $4 \mathbf{j}$ & $98.4 \pm 0.3$ & 100 & 100 & 100 & 100 & 100 & 100 & 100 & 100 & 100 & $91.1 \pm 0.6$ & $78.9 \pm 2.1$ & $56.4 \pm 2.1$ \\
\hline $4 \mathbf{k}$ & 100 & 100 & 100 & 100 & 100 & 100 & 100 & 100 & 100 & 100 & 100 & $99.7 \pm 0.2$ & $90.8 \pm 0.9$ \\
\hline Tetracaine & $98.7 \pm 0.2$ & 100 & 100 & 100 & 100 & 100 & 100 & 100 & $94.1 \pm 0.5$ & $89.6 \pm 0.7$ & $70.3 \pm 1.0$ & $46.3 \pm 3.1$ & $32.1 \pm 3.2$ \\
\hline Pramocaine & $99.1 \pm 0.2$ & 100 & 100 & 100 & 100 & 100 & 100 & $98.5 \pm 0.4$ & $90.3 \pm 0.8$ & $81.5 \pm 0.6$ & $63.9 \pm 2.1$ & $40.2 \pm 3.0$ & $25.5 \pm 3.4$ \\
\hline NS & 0 & 0 & 0 & 0 & 0 & 0 & 0 & 0 & 0 & 0 & 0 & 0 & 0 \\
\hline
\end{tabular}


Table 4 Block anesthetic effects of the target compounds and results of acute toxicity tests

\begin{tabular}{lcll}
\hline Compound & \multicolumn{2}{l}{$\begin{array}{l}\text { Onset of analgesia } \\
(\mathrm{min})\end{array}$} & $\begin{array}{l}\text { Duration of analgesia } \\
(\mathrm{min})\end{array}$ \\
\hline $\mathbf{4 d}$ & $8.8 \pm 0.4$ & $230.7 \pm 3.2$ & $543.7 \pm 2.3$ \\
$\mathbf{4 g}$ & $8.3 \pm 0.4$ & $254.6 \pm 3.6$ & $854.6 \pm 3.7$ \\
$\mathbf{4 j}$ & $12.5 \pm 0.7$ & $221.8 \pm 3.0$ & $735.1 \pm 3.2$ \\
$\mathbf{4 k}$ & $7.2 \pm 0.3$ & $279.9 \pm 3.2$ & $907.2 \pm 4.3$ \\
$\mathbf{4 n}$ & $6.4 \pm 0.2$ & $293.5 \pm 3.4$ & $976.5 \pm 4.5$ \\
$\mathbf{4 0}$ & $6.6 \pm 0.2$ & $286.4 \pm 3.3$ & $928.9 \pm 4.9$ \\
Tetracaine & $12.3 \pm 0.9$ & $172.3 \pm 2.6$ & $435.4 \pm 3.3$ \\
Pramocaine & $10.4 \pm 0.8$ & $166.5 \pm 2.3$ & $472.1 \pm 3.6$
\end{tabular}

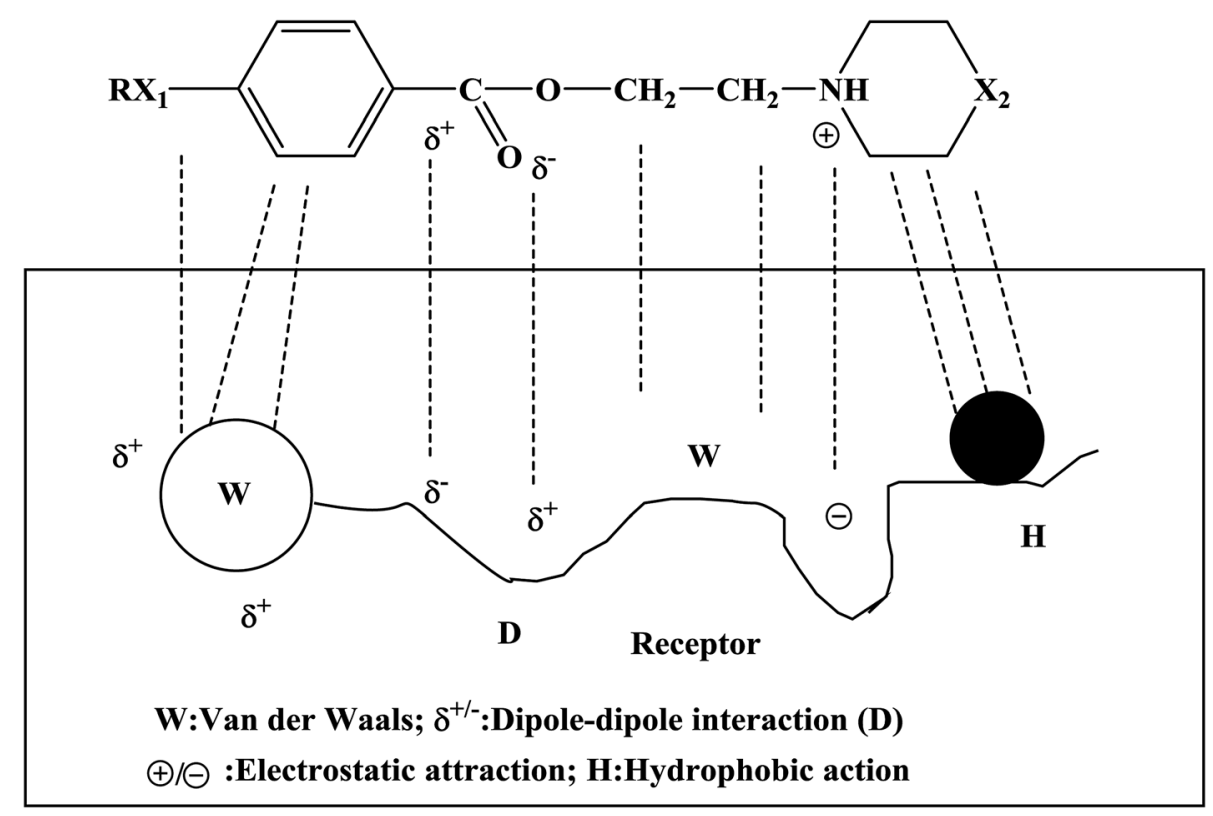

Fig. 4 Binding of the target compounds to the receptor.

compounds $\mathbf{4 k}, \mathbf{4 n}$ and $\mathbf{4 o}$ had the characteristics of rapid onset and long duration. In addition, the results of the acute toxicity test showed that the median lethal dose $\left(\mathrm{LD}_{50}\right)$ of compounds 4k, 4n and $4 \mathbf{0}$ was higher and indicated that the target compounds had low toxicity. In the study of the mechanism of local anesthetic action, we found that the $\mathrm{Na}^{+}$channels bound to by this kind of drug bind to the receptor, and thus the passage of $\mathrm{Na}^{+}$was affected. The binding of these compounds to the receptor involves multiple bonds (Fig. 4), including van der Waals forces, dipole-dipole interactions, electrostatic attraction, and hydrophobic action.

\section{Conclusion}

Tetracaine and pramocaine were used as the lead compounds, and a total of 16 target compounds were designed and synthesized. The combination principle was used to design the target molecule, which was modified by bioisostere formation and modification with alkyl groups to design benzoate compounds. We selected three steps for the synthesis of the target compounds via a route with high total yields, mild conditions and simple operation.

The biological activity was evaluated by surface anesthesia, infiltration anesthesia, block anesthesia and acute toxicity tests, and the experimental results showed that compounds $\mathbf{4 d}, \mathbf{4 g}, \mathbf{4 j}$, $\mathbf{4 k}, \mathbf{4 n}$, and $4 \mathbf{o}$ had a good local anesthetic effect. The results of acute toxicity tests showed that the target compounds had low toxicity.

\section{Conflicts of interest}

The authors declare that they have no conflict of interest.

\section{Acknowledgements}

The Project was sponsored by the Innovation Team Project of Universities in Chongqing (No. CXTDX201601018). The Project was also supported by the Chongqing Scientific and Technological Innovation Special Project of Social Undertakings and People's Livelihood Guarantee (No. cstc2015shmszx80060), Chongqing University Students' Training Project of Innovation 
and Undertaking (201510637085), Doctoral Program of Chongqing Normal University (No. 12XLB006), Outstanding Achievements Transformation Project in Chongqing Normal University (No. 15XZH08), National Natural Science Foundation (No. 216602012, No. 41866005), and Postgraduate Research and Innovation Project of Hainan Normal University (Hsyx2018-8). All animal procedures were performed in accordance with the Guidelines for Care and Use of Laboratory Animals of Chongqing Institute of Chinese Materia Medica, and the experiments were approved by the Animal Ethics Committee of Chongqing Institute of Chinese Materia Medica.

\section{References}

1 A. Brown, R. Weiss, C. Greenberg, et al., Interscalene block for shoulder arthroscopy: comparison with general anesthesia, Arthroscopy, 1993, 9(3), 295-300.

2 G. Weinberg, T. VadeBoncouer, G. Ramaraju, et al., Pretreatment or resuscitation with a lipid infusion shifts the dose-response to bupivacaine-induced asystole in rats, Anesthesiology, 1998, 88(4), 1071-1075.

3 G. Weinberg, R. Ripper, D. Feinstein, et al., Lipid emulsion infusion rescues dogs from bupivacaine-induced cardiac toxicity, Reg. Anesth. Pain Med., 2003, 28(3), 198-199.

$4 \mathrm{~J}$. Picard and T. Meek, Lipid emulsion to treat overdose of local anaesthetic: the gift of the glob, Anaesthesia, 2006, 61(2), 107-109.

5 M. Rosenblatt, M. Abel, G. Fischer, et al., Successful use of a $20 \%$ lipid emulsion to resuscitate a patient after a presumed bupivacaine-related cardiac arrest, Anesthesiology, 2006, 105(1), 217-225.

6 R. Litz, M. Popp, S. Stehr, et al., Successful resuscitation of a patient with ropivacaine-induced asystole after axillary plexus block using lipid infusion, Anaesthesia, 2006, 61(8), 800-801.

7 G. Cave and M. Harvey, Intravenous lipid emulsion as antidote beyond local anesthetic toxicity: a systematic review, Acad. Emerg. Med., 2009, 16(9), 815-824.

8 A. Sirianni, K. Osterhoudt, D. Calello, et al., Use of lipid emulsion in the resuscitation of a patient with prolonged cardiovascular collapse after overdose of bupropion and lamotrigine, Acad. Emerg. Med., 2008, 51(4), 412-415.

9 M. Harvey and G. Cave, Intralipid outperforms sodium bicarbonate in a rabbit model of clomipramine toxicity, Acad. Emerg. Med., 2007, 49(2), 178-185.

10 M. Harvey, G. Cave, K. Hoggett, et al., Correlation of plasma and peritoneal diasylate clomipramine concentration with hemodynamic recovery after intralipid infusion in rabbits, Acad. Emerg. Med., 2009, 16(2), 151-156.

11 D. Crandell and G. Weinberg, Moxidectin toxicosis in a puppy successfully treated with intravenous lipids, J. Vet. Emerg. Crit. Care, 2009, 19(2), 181-186.

12 S. Kampe, M. Warm, S. Kasper, et al., Concept for postoperative analgesia after pedicled TRAM flaps: Continuous wound instillation with $0.2 \%$ ropivacaine via multilumen catheters. A report of two cases, Br. J. Plast. Surg., 2003, 56(5), 478-483.
13 L. Nielsen, P. Lumholt and L. Halmich, Local anaesthesia with vasoconstrictor is safe to use in areas with endarteries in fingers, toes, noses and ears, Ugeskr. Laeg., 2014, 176(44), 44.

14 S. Györke and V. Lukyanenko, Dual effects of tetracaine on spontaneous sodium release in rat ventricular myocytes, $J$. Physiol., 1997, 500(2), 297-309.

15 S. Derry, P. Wiffen, R. Moore, et al., Topical lidocaine for neuropathic pain in adults, Cochrane Database Syst. Rev., 2014, 7, CD010958.

16 A. Martí, D. Simancas, V. Anand, et al., Prophylactic lidocaine for myocardial infarction, Cochrane Database Syst. Rev., 2015, 8, CD008553.

17 L. Slaughter, A. Patel and J. Slaughter, Pharmacological treatment of neonatal seizures: a systematic review, J. Child Neurol., 2013, 28(3), 351-364.

18 R. Morabito, A. Marino, S. Dossena, et al., Nematocyst discharge in Pelagia noctiluca (Cnidaria, Scyphozoa) oral arms can be affected by lidocaine, ethanol, ammonia and acetic acid, Toxicon, 2014, 83, 52-58.

19 M. Segal, G. Rogers, H. Needleman, et al., Hypokalemic sensory overstimulation, J. Child Neurol., 2007, 22(12), 1408-1410.

20 D. Jackson, A. Chen and C. Bennett, Identifying true lidocaine allergy, J. Am. Dent. Assoc., 1994, 125(10), 13621366.

21 J. Picard, S. Ward, R. Zumpe, et al., Guidelines and the adoption of 'lipid rescue' therapy for local anaesthetic toxicity, Anaesthesia, 2009, 64(2), 122-125.

22 A. Gulihar, S. Robati, H. Twaij, et al., Articular cartilage and local anaesthetic: A systematic review of the current literature, J. Orthop., 2015, 12, S200-S210.

23 W. A. Carterall, Molecular mechanisms of gating and drug block of sodium channels, Sodium Channels and Neuronal Hyperexcitability, Novartis Foundation Symposia, 2001, vol. 241, pp. 206-225.

24 S. Sheu and W. Lederer, Lidocaine's negative inotropic and antiarrhythmic actions. Dependence on shortening of action potential duration and reduction of intracellular sodium activity, Circ. Res., 1985, 57(4), 578-590.

25 K. Collinsworth, S. Kalman and D. Harrison, The clinical pharmacology of lidocaine as an antiarrhythymic drug, Circulation, 1974, 50(6), 1217-1230.

26 A. Pupka, J. Sikora, J. Mauricz, et al., The usage of synthol in the body building, Polim. Med., 2009, 39(1), 63-65.

27 N. Bernardo, M. Siqueira, M. DePaiva, et al., Caffeine and other adulterants in seizures of street cocaine in Brazil, Int. J. Drug Policy, 2003, 14(4), 331-334.

28 A. Gargiulo, G. Burns and C. Huck, Dyclonine hydrochloridea topical agent for managing pain, Ill. Dent. J., 1992, 61(4), 303-304.

29 J. Schmidt, L. Blockus and R. Richards, The Pharmacology of Pramoxine Hydrochloride: A New Topical Local Anesthetic, Curr. Res. Anesth. Analg., 1953, 32(61), 418-425. 